\title{
CARACTERIZACIÓN DE PROPIEDADES MECÁNICAS PARA SEGREGAR FAMILIAS DE Eucalyptus nitens POR NANOINDENTACIÓN EN RELACIÓN AL GRADO DE AGRIETAMIENTO DE LAS TROZAS
}

\section{MECHANICAL PROPERTIES CHARACTERIZATION TO SEGREGATE OF Eucalyptus nitens FAMILIES BY NANOINDENTATION RELATED TO THE DEGREE OF CRACKING IN LOGS}

\author{
Paulina Valenzuela ${ }^{1, \wedge}$, Cecilia Bustos $^{2}$, Jean Pierre Lasserre³, William Gacitúa ${ }^{2}$
}

\section{RESUMEN}

Seis familias de Eucalyptus nitens provenientes de dos suelos, arcilloso y trumao, localizados en la $8^{\text {va }}$ Región de Chile, fueron estudiadas para seleccionar aquellas familias con menor grado de agrietamiento. Se relacionaron parámetros de agrietamiento evaluados a mesoescala con las propiedades nanomecánicas en un elemento de volumen representativo de estos árboles. Para segregar las familias se analizó el porcentaje de área de grietas de estos árboles junto a la razón de ductilidad $(E / H)$ obtenidos del módulo de elasticidad $(E)$ y dureza $(H)$ mediante la técnica de nanoindentación y el módulo de resiliencia $\left(u_{r}\right)$ ambos en la lamela media y capa S2 de la pared celular. Además, se determinó la diferencia entre el módulo de resiliencia entre la lamela media y la capa $\mathrm{S}_{2}(\Delta u r)$, como un indicador de la tensión interfacial que induce fracturas principalmente en la capa $\mathrm{S}_{1}$ de la estructura celular de la madera. La nanocaracterización permitió establecer que la lamela media es más débil y susceptible a generar microgrietas que se propagan hasta ser visibles formando grietas mayores. El parámetro $\Delta$ ur entre la lamela media y capa $S_{2}$ mostró ser menor en las familias 2 y 5 . Estas familias presentaron menores niveles de tensión de corte en la interface de la capa $\mathrm{S}_{2}$-lamela media, por lo tanto, la cantidad de microgrietas, existiendo una buena relación con el porcentaje de grietas medidos en las familias de Eucalyptus nitens. De acuerdo a esto, las familias segregadas con bajo grado de tensión interfacial a nivel celular y bajo nivel de agrietamiento a mesoescala, fueron las familia 2 y 5 .

Palabras claves: Eucalyptus nitens, grietas internas, nanoindentaciones, pared celular, propiedades mecánicas.

\begin{abstract}
Six Eucalyptus nitens families from two soils in the $8^{\text {th }}$ Region of Chile, clay and trumao, were studied to select families with lower degree of cracking. Cracking parameters evaluated at mesoscale, next to nanomechanical properties of the celular structure, were related in a representative volumen element of these tree, such as percentage of cracked area of trees relating them to the ductility ratio $(E / H)$ obtained of values of modulus of elasticity $(E)$ and hardness $(H)$ by nanoindentation and the modulus of resilience $\left(u_{r}\right)$ of the middle lamella and $\mathrm{S}_{2}$-layer of the cell wall were analyzed. Furthermore, it was determined that the difference between the modulus of resilience $\left(\Delta u_{r}\right)$ of the middle lamella with the $\mathrm{S}_{2}$-layer is a good indicator of the interfacial stress inducing fractures in the $\mathrm{S}_{1}$-layer. Using nanocharacterization it was established that the middle lamella is more brittle and susceptible to generate microcracks that spread to meso scale. The $\Delta u_{r}$
\end{abstract}

\footnotetext{
${ }^{1}$ Investigadora del Centro de Biomateriales y Nanotecnología (CBN). Universidad del Bío-Bío. Concepción, Chile.

${ }^{2}$ Profesor asociado. Centro de Biomateriales y Nanotecnología (CBN). Depto. Ingeniería en Maderas, Facultad de Ingeniería.

Universidad del Bío-Bío. Concepción, Chile.

${ }_{3}^{3}$ CMPC Forestal Mininco S.A. Concepción, Chile.

^Autor para correspondencia: nvalenzu@ubiobio.cl

Recibido: 06.05.2014 Aceptado: 03.11.2014
} 
between the middle lamella and $\mathrm{S}_{2}$ layer shown to be lower in families 2 and 5 . These families showed to have lower levels of shear stress at the interface between the middle lamella and $\mathrm{S}_{2}$-layer, therefore the amount of microcracks that occur is lower, there is a good relationship with the percentage of measured cracks in these families of Eucalyptus nitens. According these findings, the families that show low degree of interfacial tensión at celular level and low cracks index were family 2 and family 5.

Keywords: Cracks, Eucalyptus nitens, nanoindentations, mechanical properties, wood cell-wall.

\section{INTRODUCCIÓN}

La especie de Eucalyptus nitens fue introducida en Chile para conocer las características y comportamientos en suelos nacionales, demostrando poseer grandes ventajas, causando un gran interés en la industria forestal. Desde hace un tiempo, grupos de investigación han realizado estudios de propiedades físico-mecánicas, químicas, trabajabilidad y secado para demostrar que esta especie puede otorgar grandes oportunidades en los mercados mundiales de la madera (INFOR 2004a). Sin embargo, el potencial del Eucalyptus nitens se enfoca principalmente en la producción de pulpa (Smethurst et al. 2003, INFOR 2004b) dada las ventajas que presenta, tales como la adaptabilidad y resistencia a las bajas temperaturas de hasta $-10{ }^{\circ} \mathrm{C}$ y una tasa de crecimiento de 30 a $45 \mathrm{~m}^{3} /$ ha-año, registrándose en la décima región de Chile incluso incrementos de hasta $70 \mathrm{~m}^{3} /$ ha-año (INFOR 2004b, Pinkard y Beadle 1998, Valencia y Cabrera 2003). No obstante, el rendimiento pulpable del Eucalyptus nitens es menor en comparación a otras especies como el Eucalyptus globulus, dada la baja densidad que esta especie presenta, siendo poco adecuada para los actuales sistemas de pulpaje (Valencia y Cabrera 2003). Es así como nace una búsqueda constante de los factores que influyen en la calidad de la madera para darle un uso estructural a esta especie, de manera tal de otorgarle mayor valor agregado (Raymond y Apiolaza 2004, Bravo et al. 2012, Medhurst et al. 2001, Nolan et al. 2005, Baker y Volker 2007, Acevedo et al. 2012, Forrester et al. 2010). Sin embargo, la desventaja del Eucalyptus nitens radica fundamentalmente en la formación de grietas que aparecen luego de la tala de los árboles, concediéndole un bajo nivel de aprovechamiento en potenciales productos de ingeniería.

La formación de las grietas se debe a tensiones internas que se producen en el proceso de crecimiento del árbol, las que se inician en el cambium. Las células que se producen en el cambium, en su proceso de crecimiento, tienden a expandirse lateralmente y a contraerse longitudinalmente, lo que es impedido por las células formadas en años anteriores, generando un conjunto de tensiones que se liberan generalmente con la tala del árbol (Vignote et al. 1996, Touza 2001, Smith et al. 2003). Para aumentar la productividad y la calidad de los árboles debido a la desventaja del Eucalyptus nitens, se ha trabajado en el mejoramiento genético y en la selección de los mejores árboles (Raymond 2002, Tibbits y Hodge 1998, Greaves et al. 1996, Rezende et al. 2014), para ser reproducidos con destacables características de crecimiento y forma.

Por otro lado, el tipo de suelo es otro factor que influye en el crecimiento y calidad de los árboles. El desarrollo del Eucalyptus nitens se ha visto favorecido principalmente en suelos arcillosos profundos, aún cuando se han observado interesantes crecimientos en suelos de tipo trumao (INFOR 2004b).

Plantaciones con mayores edades intervenidas genéticamente pueden ser segregadas y utilizadas en el desarrollo de productos de ingeniería, según sus características mecánicas a nanoescala en la estructura celular, donde se originarían las microgrietas (Gacitúa et al. 2007, Côte y Hanna 1983). Un método no destructivo para obtener estas propiedades nanomecánicas es la técnica de nanoindentación. Esta técnica ha sido utilizada para investigar el comportamiento de la madera o composite (Wu et al. 2009, Muñoz et al. 2012, Valenzuela et al. 2012a, Konnerth y Gindl 2006, Bustos et al. 2011). Otra característica que puede ser considerada para segregar árboles Eucalyptus nitens es la frecuencia de grietas debido a que se presentan de acuerdo a las características del árbol (Valenzuela 2012b, Leandro et al. 2008). 
El objetivo de este estudio fue utilizar la técnica de nanoindentación para segregar familias de Eucalyptus nitens, desde dos sitios con distintos suelos, de acuerdo a su grado de agrietamiento y a las propiedades nanomecánicas de la madera, con potencial para fabricar productos de ingeniería tal como tableros contrachapados.

\section{MATERIALES Y MÉTODOS}

\section{Madera}

Se utilizaron 6 familias de Eucalyptus nitens (Deane and Maiden) de 12 años de edad provenientes de dos sitios con distinto tipo de suelo, ubicados en la VIII región de Chile. La ubicación de estos sitios corresponden

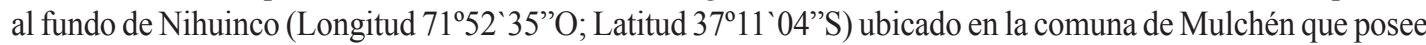

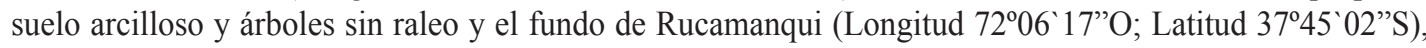
comuna de Yungay correspondiendo a suelo trumao y a un bosque con raleo. El espaciamiento en ambas plantaciones fue de $2 \times 3 \mathrm{~m}$. Finalmente, se estudiaron un total de 12 genotipos. Familia se les llama a los genotipos que están emparentados por al menos un progenitor común (madre). La procedencia de las semillas de cada familia, se obtuvieron en distintas zonas geográficas de Australia (Hamilton et al. 2008), y la fuente natural de la población se detalla en la tabla 1.

Tabla 1. Procedencia de familias de E. nitens localizadas en fundo Nihuinco y Rucamanqui, VIII región, Chile.

\begin{tabular}{|c|l|l|}
\hline Código Familia & \multicolumn{1}{|c|}{ Origen } & \multicolumn{1}{c|}{ Raza } \\
\hline 1 & Toorongo Plateau & SouthCentVic \\
\hline 2 & Toorongo & SouthCentVic \\
\hline 3 & Macalister & NorthCentVic \\
\hline 4 & MT Tangle foot & SouthCentVic \\
\hline 5 & Rubicon Snobs CK Rd & NorthCentVic \\
\hline 6 & Rubicon & NorthCentVic \\
\hline
\end{tabular}

Para las seis familias de ambos tipos de suelos, se determinaron las propiedades nanomecánicas de la estructura celular y el porcentaje de grietas en semi-rodelas.

\section{Nanoindentaciones}

Las probetas para el análisis de propiedades a nanoescala se obtuvieron del elemento de volumen representativo, a una altura de 3 metros desde la base del árbol, y en los anillos de crecimiento 5, 6 y 7 desde la médula hacia la corteza, según estudio de Valenzuela et al. (2012b).

De cada árbol se cortó un disco de 2 pulgadas de espesor, cortándola en el centro para obtener un listón de $1 \mathrm{~cm}$ de ancho, utilizando sólo el hemisferio norte debido a que se presentan las mayores fuerzas externas que actúan en los árboles, y por lo tanto mayor frecuencia de grietas. De cada listón, se obtuvieron 3 cubos (anillos de crecimiento 5, 6 y 7) de $3 \mathrm{~mm}$ de lado en la zona de madera de primavera donde se generan principalmente las grietas en el Eucalyptus nitens (Gacitúa et al. 2007). Los cubos fueron impregnados con resina epóxica para brindarle mayor soporte mecánico a la estructura celular en los ensayos de nanoindentación y evitar el daño mecánico que se puede producir al realizar cortes con el micrótomo (Jakes et al. 2007). En un micrótomo de rotación Leica RM2265 se cortaron láminas de Eucalyptus nitens de $250 \mathrm{~nm}$ de espesor, usando un cuchillo de vidrio y luego uno de diamante para conseguir que la superficie de cada cubo quedara completamente lisa y uniforme, para las mediciones de las propiedades mecánicas a nanoescala. Las láminas fueron cortadas en el plano transversal del cubo, quedando expuesta la sección transversal de las fibras. Los cubos fueron acondicionados por un tiempo no menor a $24 \mathrm{~h} \mathrm{a} 21^{\circ} \mathrm{C}$ y a una humedad relativa de $60 \%$. Las propiedades 
a nanoescala fueron medidas con un nanoindentador Hysitron TI900. Se utilizó una punta de diamante tipo cube corner y se aplicó una carga nominal máxima de $100 \mu \mathrm{N}$. Previo a los ensayos, el equipo fue calibrado de acuerdo a las especificaciones técnicas del fabricante del equipo, a través del método de función de área, lo cual permite asegurar que los valores del módulo de elasticidad $(E)$ y la dureza $(H)$ sean los correctos.

La figura 1 muestra las marcas dejadas por esta punta de diamante luego de un ensayo de nanoindentaciones en la capa $\mathrm{S}_{2}$ y en la lamela media (LM), con las cuales se obtienen los valores de $E$ y $H$. De cada anillo de crecimiento (5, 6 y 7), se realizaron nanoindentaciones en 3 zonas. En estas 3 zonas, se realizaron 6 nanoindentaciones. De esta forma, se realizaron un total de 54 nanoindentaciones por familia.

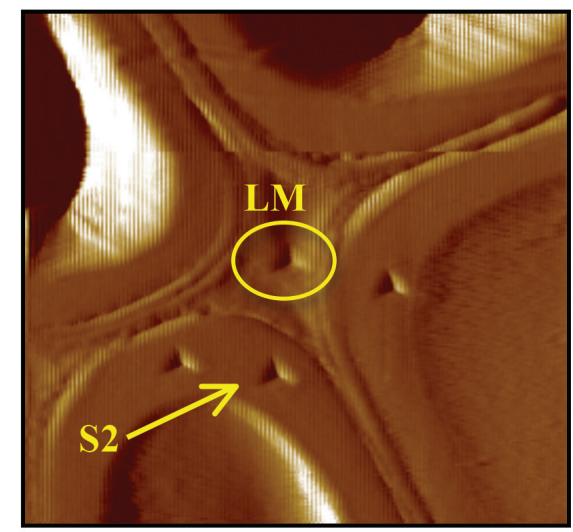

Figura 1. Imagen en 2D post-ensayo de nanoindentaciones en células de Eucalyptus nitens en capa $\mathrm{S}_{2}$ y lamela media (LM)

(Área de la imagen: 10x10 $\mu \mathrm{m}$ ).

Se diseñó un ciclo de carga con un segmento de carga constante para evitar la influencia del creep, tanto en la capa $\mathrm{S}_{2}$ como en la LM. El ciclo comenzó con un tiempo de carga de $5 \mathrm{~s}$, que se mantuvo constante por $60 \mathrm{~s}$ y finalmente una descarga en $5 \mathrm{~s}$. De esta manera, la medición en la descarga donde se produce la deformación elástica, no incluye comportamientos viscoelásticos en las respuestas medidas (Zou et al. 2009, Wimmer y Lucas 1997).

Se determinó la razón de ductilidad $(E / H)$ y el módulo de resiliencia $\left(u_{r}\right)$, a través del módulo de elasticidad $(E)$ de la muestra obtenida por la ecuación del módulo elástico reducido $\left(E_{r}\right)$ (Oliver y Pharr 1992):

$$
\frac{1}{E_{r}}=\frac{1-v^{2}}{E}+\frac{1-v_{i}^{2}}{E_{i}}
$$

Donde $E$ y $v$ son el módulo de elasticidad y razón de poisson del material en estudio; en este caso capa $\mathrm{S}_{2}$ o LM. Por otro lado, $E_{i}$ y $v_{i}$ son los mismos parámetros conocidos para la punta de diamante (indentador).

La dureza se determinó usando la ecuación 2 (Oliver y Pharr 1992):

$$
H=\frac{P_{\max }}{A}
$$

Donde $P_{\max }$ es la carga medida en la máxima penetración del indentador y $A$ es el área proyectada o de contacto en la carga máxima. 
El módulo de resiliencia $\left(u_{r}\right)$ (ecuación 3), es el área que se encuentra bajo la curva esfuerzo-deformación, hasta el esfuerzo en el límite proporcional $\left(\sigma_{y}\right)$ (Figura 2), y representa la energía que puede ser absorbida por un material por unidad de volumen sin superar el límite elástico y sin sufrir deformación permanente (Ferdinand y Rusell 1992, Hibbeler 2006).

$$
u_{r}=\frac{\sigma_{y}^{2}}{2 E}
$$

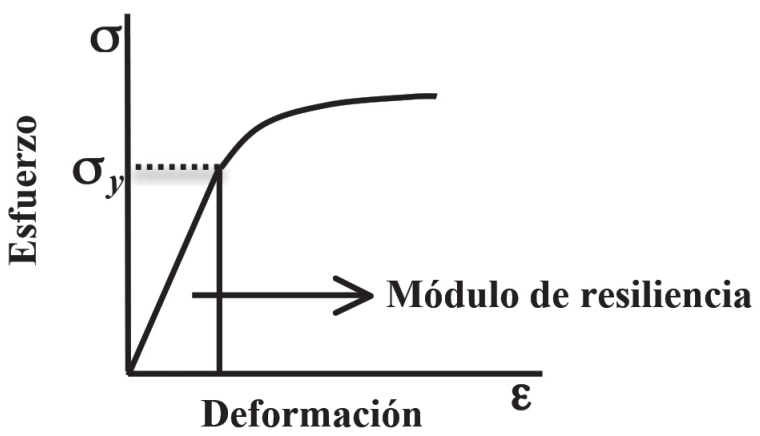

Figura 2. Módulo de resiliencia, área bajo la curva de esfuerzo y deformación.

Otra variable respuesta evaluada es la diferencia entre el módulo de resiliencia de la lamela media y el módulo de resiliencia de la capa $\mathrm{S}_{2}$ (delta módulo de resiliencia: $\Delta u_{r}$ ). Se postula a que si este parámetro aumenta, indicaría que las tensiones en la interface también aumentarían generando tensiones de corte en la capa $\mathrm{S}_{1}$. Para esto, se utilizaron los datos de los módulos de resiliencia de la capa $\mathrm{S}_{2}$ y LM, obteniendo seis datos promedios por cada familia.

\section{Análisis estadístico nanoindentaciones}

Para las propiedades nanomecánicas, el análisis de Varianza (ANOVA) se analizó en el software Statgraphics Centurion XV V15.2, con un diseño multifactorial considerando los factores: suelo (2 niveles: arcilloso y trumao), familia (6 niveles: $1,2,3,4,5$ y 6) y tipo de material (capa $\mathrm{S}_{2}$ y LM). En el caso de la diferencia del módulo de resiliencia entre la LM y la capa $S_{2}$ se utilizó un diseño factorial general considerando los factores: suelo (2 niveles: arcilloso y trumao) y familia (6 niveles: 1, 2, 3, 4, 5 y 6). Los datos fueron analizados con un nivel de confianza del $95 \%$. Para el análisis de las superficies respuesta se utilizó el software Design Expert V8.0.

\section{Medición de grietas}

El porcentaje de grietas del Eucalyptus nitens, se midió en la mitad de las rodelas (semi-rodelas) cortadas a 1, 2 y 3 metros de la altura de los árboles. Se utilizaron 4 datos; dos datos a la altura del metro 1 y 2 , y dos datos a la altura del metro 3. Estas alturas fueron seleccionadas en base a un estudio que reporta mayor frecuencia de grietas del Eucalyptus nitens en los primeros 3 metros de altura del árbol (Leandro et al. 2008), y a una investigación que reporta como altura representativa a la altura en el metro 3 (Valenzuela et al. 2012b). La semi-rodela incluyó tanto el lado norte como el lado sur (Figura 3). Se asumió comportamiento simétrico de las grietas en ambas semi-rodelas. Las semi-rodelas se dejaron secar al aire por un período de 30 días a temperatura ambiente (promedio de $20^{\circ} \mathrm{C}$ ) y humedad relativa de $65 \%$ para estudiar el agrietamiento. Se capturaron imágenes de las semi-rodelas con una cámara fotográfica digital Panasonic DMC FZ8. Antes de realizar las mediciones de grietas, las imágenes fueron tratadas con el software Image-Pro plus, mejorando el contorno y visibilidad de las grietas para disminuir el margen de error en las mediciones. Se midió el porcentaje de área de las grietas (semi-rodela) con respecto del área total evaluada. Se obtuvieron cuatro datos; un dato 
en el metro 1 y 2 y dos datos en el metro 3 , se consideraron dos datos porque fue la altura representativa según estudio realizado por Valenzuela et al. (2012b)

\section{Análisis estadístico medición de grietas}

El análisis estadístico se realizó con el software Design Expert V8.0 a través de un Análisis de Varianza (ANOVA), con un diseño factorial general considerando los factores: suelo (2 niveles: arcilloso y trumao) y familia (6 niveles: 1, 2, 3, 4, 5 y 6). Los datos fueron analizados con un nivel de confianza del 95\%.

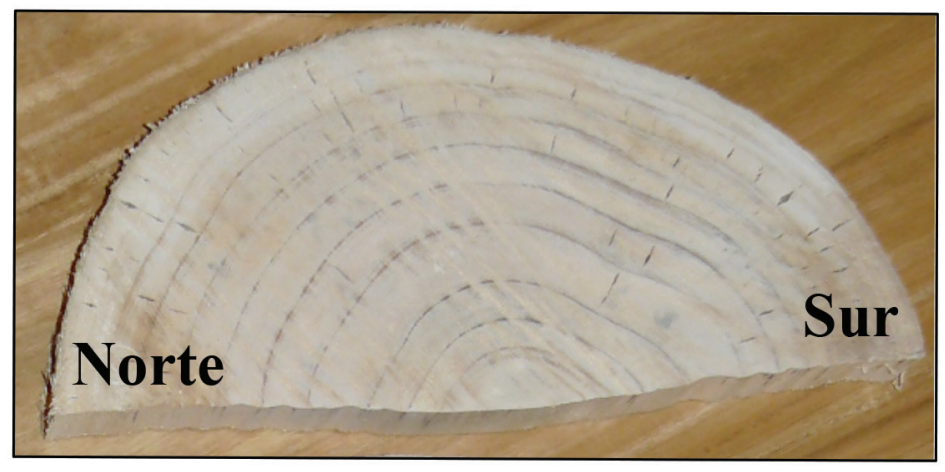

Figura 3. Orientación Norte-Sur en rodela de Eucalyptus nitens usada para medición de grietas.

\section{RESULTADOS Y ANÁLISIS}

La tabla 2 muestra el valor- $p$ obtenido en ANOVA, indicando si son o no significativos los factores e interacciones para el modelo y las variables respuestas. Los resultados muestran que el modelo y los tres factores suelo, familia y tipo de material presentaron un efecto significativo en ambas variables respuesta $\left(E / H\right.$ y $\left.u_{r}\right)$. La variable respuesta $E / H$ mostró un efecto significativo en la interacción de segundo y tercer orden contribuyendo con esto al modelo. Para la variable respuesta $u_{r}$ sólo mostró un efecto significativo la interacción de segundo orden $\mathrm{AB}$ (sitio-familia). El análisis de residuales muestra que los datos cumplen con los supuestos de normalidad y varianza constante. Por otro lado, los valores de $\mathrm{R}^{2} \mathrm{y}^{2}$-ajustado señalan que los factores presentados en el modelo son apropiados, confirmando la validez del ANOVA.

Tabla 2. Valor $-p$ de variables respuestas, ANOVA.

\begin{tabular}{|c|c|c|}
\hline \multicolumn{3}{|c|}{ Valor-p $*(0,05)$} \\
\hline Factor & $E / H$ & $u_{r}$ \\
\hline Modelo & $<0,0001$ & $<0,0001$ \\
\hline A-Suelo & 0,0411 & 0,0027 \\
\hline B-Familia & $<0,0001$ & 0,0028 \\
\hline C-Tipo Material & $<0,0001$ & $<0,0001$ \\
\hline $\mathrm{AB}$ & $<0,0001$ & $<0,0001$ \\
\hline $\mathrm{AC}$ & $<0,0001$ & 0,0778 \\
\hline $\mathrm{BC}$ & $<0,0003$ & 0,3763 \\
\hline $\mathrm{ABC}$ & $<0,0001$ & 0,7454 \\
\hline $\mathrm{R}^{2}$ & 0,8836 & 0,9225 \\
\hline $\mathrm{R}^{2}$ ajustado & 0,8814 & 0,9074 \\
\hline
\end{tabular}

$E / H$ : razón de ductilidad. $u_{r}$ : modulo de resiliencia. Suelo: arcilloso y trumao. Familia: 1, 2, 3, 4, 5 y 6 . Tipo de material: capa $\mathrm{S}_{2}$ y LM. * Valor- $p<0,05$ indica que los factores son significativos y Valor- $p>0,05$ indica que lo factores no son significativos. 

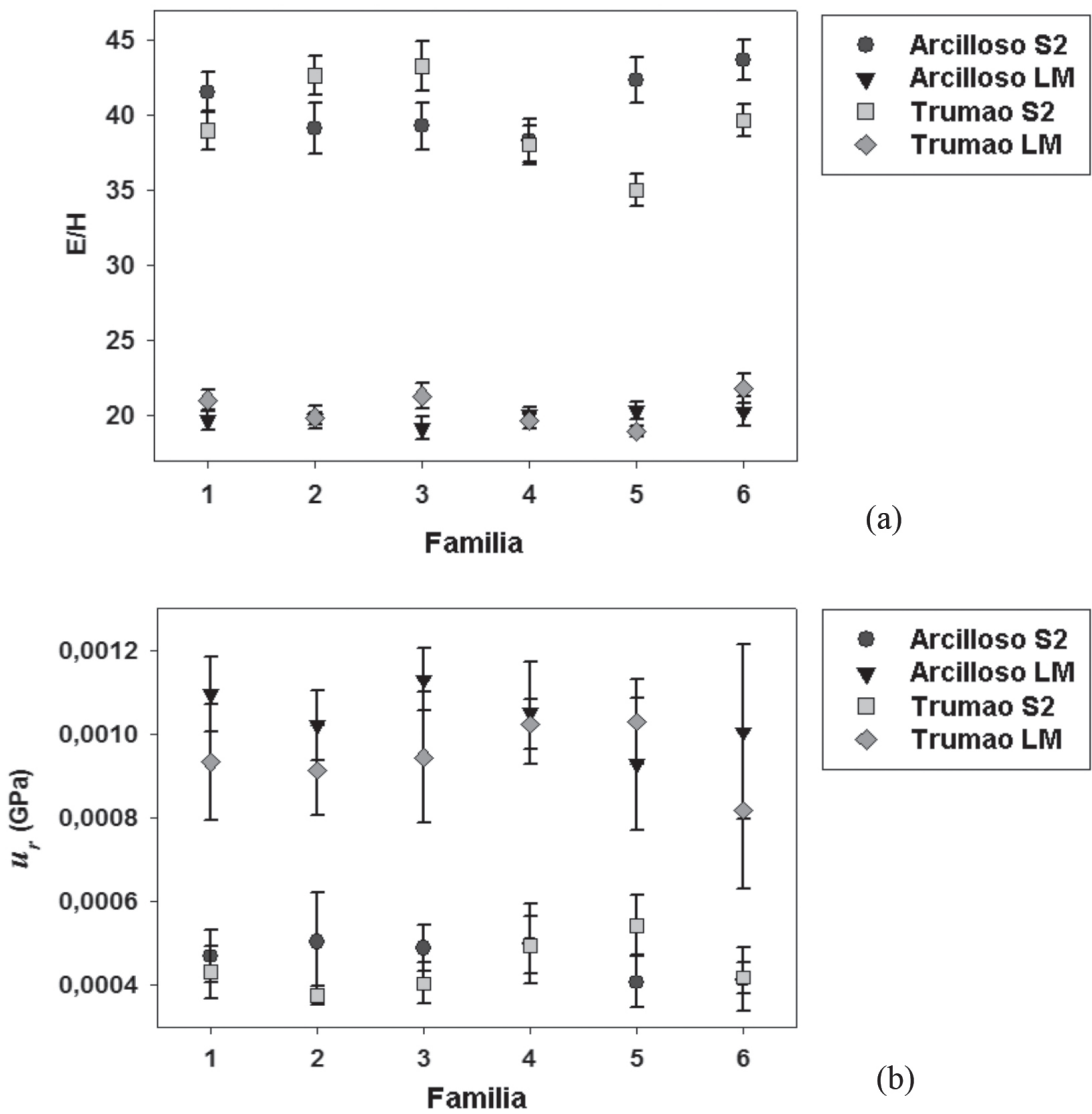

Figura 4. Propiedades nanomecánicas de familias de Eucalyptus nitens para capa $\mathrm{S}_{2}$ y lamela media en suelos arcilloso y trumao. (a) Razón de ductilidad $E / H$ (adimensional) (b) Módulo de resiliencia $\left(u_{r}\right)$.

La figura 4 muestra el efecto entre suelos, familias y tipo de material para las propiedades nanomecánicas razón de ductilidad y módulo de resiliencia. La razón de ductilidad (Figura 4a) muestra en ambos tipos de suelos, el mismo comportamiento entre la capa $\mathrm{S}_{2}$ y la LM. La capa $\mathrm{S}_{2}$ presentó valores promedio de $E / H=$ 40,73 en suelos arcillosos y $E / H=39,63$ en suelos trumao, valores por sobre los obtenidos por la $L M$ con valores promedios de $E / H=20,29$ en suelos arcillosos y de $E / H=20,45$ para suelo trumao; esto indica que la LM tiene un comportamiento más frágil, según lo reportado por Bolshakov y Pharr (1998), quienes analizaron dos materiales con durezas distintas, el aluminio y el vidrio. Este último, con una alta dureza presentó una razón $E / H$ significativamente menor que el aluminio; materiales dúctiles tienen una alta razón de ductilidad, y por el contrario, materiales frágiles presentan una baja razón, tal como se ejemplifica con el aluminio y el vidrio. Lo contrario sucede con la capa $\mathrm{S}_{2}$ que es más dúctil, lo que le permite soportar mayores deformaciones asociadas a las tensiones de crecimiento. La diferencia en $E / H$ entre la capa $\mathrm{S}_{2}$ y la LM generaría esfuerzos de corte que provocarían la separación y formación de microgrietas (Valenzuela et al. 2012a). Putoczki et al. (2007), argumentan que cuando dos capas constituidas por materiales distintos son sometidas a cierta carga, solo una de ellas podrá deformarse elásticamente sin fracturarse dada la diferencia de ductilidad entre ellas. Esta deformación diferencial entre los materiales generaría la separación entre las capas formando las microgrietas, separación que ocurre en una capa interfacial (entre la LM y capa $\mathrm{S}_{2}$ ) llamada capa $\mathrm{S}_{1}$. 
Por otro lado, se logra observar diferencias en el tipo de suelo para la capa $\mathrm{S}_{2}$. La mayoría de las familias de Eucalyptus nitens se muestran más frágiles en suelo trumao que en suelo arcilloso. Esto puede indicar que en suelos arcillosos se generaría menor agrietamiento en los árboles, esto se relacionaría con lo reportado por INFOR, (2004b). Para este caso, las familias de Eucalyptus nitens menos dúctiles corresponderían a las familias 1, familia 5 y familia 6 del suelo arcilloso. En la LM no se observó diferencias notorias en los suelos.

El módulo de resiliencia (Figura 4b) mostró para la capa $\mathrm{S}_{2}$ valores promedio de $u_{r}=4,64 \times 10^{-4} \mathrm{GPa}$ para suelo arcilloso y de $u_{r}=4,45 \times 10^{-4} \mathrm{GPa}$ para suelo trumao, tres veces menor que la LM con un valor promedio de $u_{r}=1,04 \times 10^{-3} \mathrm{GPa}$ en suelo arcilloso y $u_{r}=9,44 \times 10^{-4} \mathrm{GPa}$ para suelo trumao. Esto indica que la LM posee la capacidad de absorber mayor cantidad de energía de deformación si se compara con la capa $\mathrm{S}_{2}$ (Gacitúa et al. 2007), ratificando de esta forma que la LM es un material resiliente y que ayuda a disipar las tensiones de crecimiento, y consecuentemente las deformaciones asociadas que pueden inducir fracturas en la estructura celular. En este sentido, se puede establecer una analogía entre la madera y un material compuesto, con una LM actuando como una matriz fuerte y rígida integrada en un reforzante flexible como las fibras compuestas por diferentes capas (Hull y Clyne 1996). La LM (matriz) puede almacenar mayor cantidad de energía permitiendo que las fibras (reforzante) se muevan sin que el material compuesto (madera) se rompa cuando es sometido a un cierto nivel de deformación. Este nivel de deformación puede ser determinado por la diferencia del módulo de resiliencia entre capas $\left(\Delta u_{r}\right)$, que en este estudio fueron la LM y la capa $\mathrm{S}_{2}$, el que se profundizará más adelante. Al observar los tipos de suelos, el arcilloso predomina tanto en capa $\mathrm{S}_{2}$ como en la LM, esto puede indicar que la estructura celular en este tipo de suelo tiene mayor capacidad de disipar energía de deformación, generando menores niveles de agrietamiento en las familias, en comparación al suelo trumao que a un cierto nivel de deformación este material sería más propenso a las fracturas. Por otro lado, las familias de Eucalyptus nitens que logran soportar mayores deformaciones en suelos arcilloso son las familia 1, familia 2 , familia 3 y familia 6 .

Como se mencionó anteriormente, la capacidad de absorción de energía de deformación que puede sufrir el material madera en un árbol, puede ser determinado en base al módulo de resiliencia $\left(u_{r}\right)$. Bajo este supuesto, se evaluó la diferencia del módulo de resiliencia $\left(\Delta u_{r}\right)$ entre la LM y la capa $\mathrm{S}_{2}$, para conocer que familias

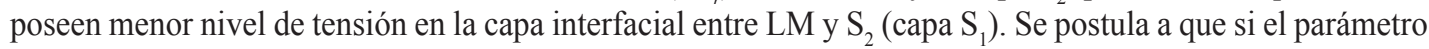
$\Delta u_{r}$ aumenta, las tensiones en la interface también aumentan, generando tensiones de corte en la capa central (capa $\mathrm{S}_{1}$ ) promoviendo aparición de las microgrietas que pueden crecer hasta ser visibles a mesoescala. Esto se relaciona con lo reportado por Gacitúa et al. (2007), Putoczki et al. (2007) y Valenzuela et al. (2012a), quienes mencionan que las microgrietas se generarían en la capa $\mathrm{S}_{1}$ y zonas cercanas a ésta.

La tabla 3 muestra los valores- $p$ obtenidos del ANOVA para los factores familia y suelo y, para las variables respuesta delta módulo de resiliencia y porcentaje de grietas. En ambas variables respuestas el modelo mostró un efecto significativo contribuyendo el factor familia, no así el factor suelo, que no mostró un efecto significativo. La variable respuesta $\Delta u_{r}$ mostró un efecto significativo en la interacción de segundo orden contribuyendo con esto al modelo, no así, el porcentaje de grietas. 
Tabla 3. Valor- $p$ de respuestas variables en ANOVA.

\begin{tabular}{lcc}
\hline \multicolumn{3}{c}{ Valor- $p^{*}(0,05)$} \\
\hline Factores & Delta Módulo de resiliencia & Porcentaje de grietas \\
\hline Modelo & $<0,0001$ & 0,0019 \\
A-familia & $<0,0001$ & 0,0010 \\
B-suelo & 0,8130 & 0,5160 \\
$\mathrm{AB}$ & $<0,0001$ & - \\
$\mathrm{R}^{2}$ & 0,5908 & 0,3937 \\
$\mathrm{R}^{2}$ ajustado & 0,5119 & 0,3028 \\
\hline
\end{tabular}

Suelo: arcilloso y trumao. Familia: 1, 2, 3, 4, 5 y 6.

* Valor- $p<0,05$ indica que los factores son significativos y Valor- $p>0,05$ indica que lo factores no son significativos.

La Figura 5 muestra el gráfico obtenido para el parámetro $\Delta u_{r}$. Según lo planteado anteriormente, se observa que las familias que muestran bajos valores de $\Delta u_{r}$, son la familia 2 y familia 5 , es decir, estos árboles presentarían menores tensiones de corte en la capa $\mathrm{S}_{1}$.

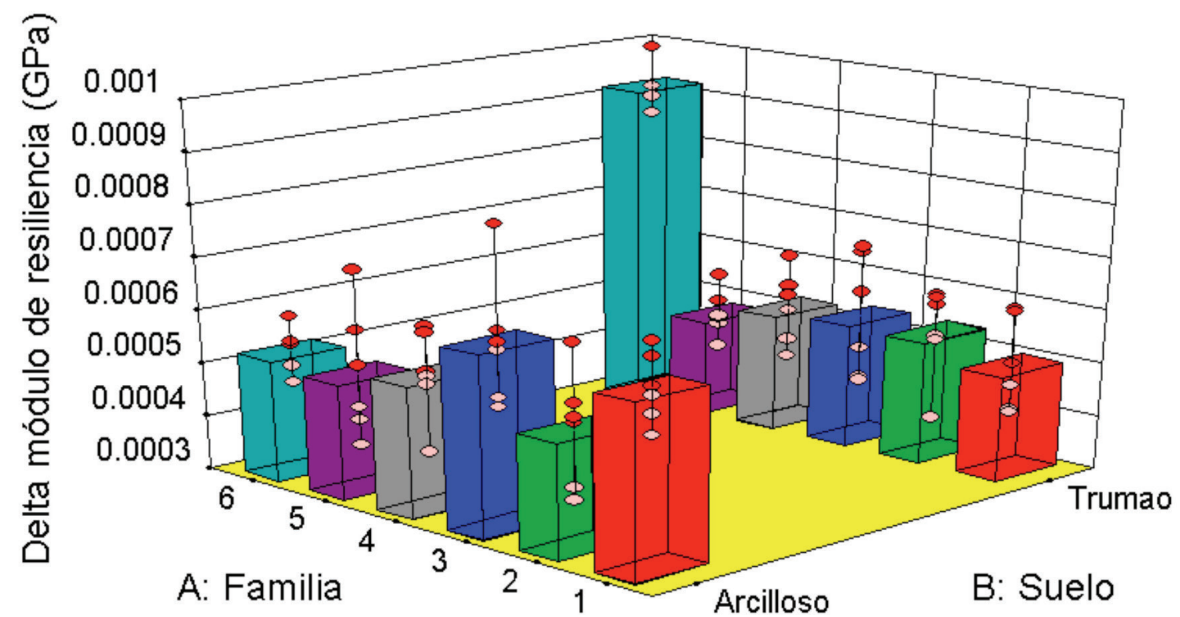

Figura 5. Diferencia del módulo de resiliencia $\left(\Delta u_{r}\right)$ entre la LM y la capa $\mathrm{S}_{2}$. Interacción suelo-familia, familias 1 a 6 . Suelo arcilloso y trumao.

Puntos indican valores individuales.

Por otro lado, la figura 6 muestra el gráfico obtenido del parámetro porcentaje de grietas. Las familias que presentaron menor porcentaje de grietas, evaluadas en las semi-rodelas, fueron la familia 2 con un valor promedio de $0,07 \%$ en suelos arcillosos y $0,25 \%$ en suelo trumao y, la familia 5 con un valor promedio de $0,13 \%$ en suelo arcilloso y $0,31 \%$ en suelo trumao. En base a estos resultados, es factible decir, que se puede asociar el parámetro $\Delta u_{r}$ a los bajos niveles de grietas presentados por estas familias de Eucalyptus nitens al evaluar el parámetro frecuencia de grietas. A la luz de estos resultados y a la tecnología de segregación mediante la relación entre propiedades nanomecánicas de la estructura celular y porcentaje de grietas medidos en zonas representativas del árbol, se puede proyectar que las familias 2 y 5 podrían tener un buen comportamiento y por lo tanto un potencial para la fabricación de productos de ingeniería en base a chapas, tal como los tableros contrachapados de Eucalyptus nitens. En paralelo a este estudio, y mediante una prueba industrial de fabricación de contrachapados con estas familias de Eucalyptus nitens segregadas, Acevedo et al. (2012), analizaron la performance de las familias segregadas para la fabricación de productos de mayor valor, particularmente tableros contrachapados. Estos autores señalan que las chapas y tableros contrachapados 
fabricados con árboles de la familia 2831 de suelos arcillosos, correspondiente a la familia 2 en este estudio, resultó tener propiedades mecánicas sobresalientes en el sentido transversal y longitudinal, con valores en el módulo de elasticidad en tracción de $E=0,10 \mathrm{GPa}$ y $E=2,25 \mathrm{GPa}$, respectivamente. Las chapas secas de las familias segregadas en este estudio, presentaron bajo nivel de agrietamiento y mejores propiedades nanomecánicas que aquellas familias susceptibles con mayor agrietamiento. De acuerdo al escalamiento de resultados de este estudio a la prueba industrial realizada por Acevedo et al. (2012), es factible establecer una relación entre el parámetro $\Delta u_{r}$ medido entre capas de la estructura celular del Eucalyptus nitens, con el porcentaje de grietas promedio a partir de muestras representativas. Para las familias con menos tendencia al agrietamiento, el $\Delta u_{r}$ es menor y para las familias con mayores tendencias al agrietamiento, $\Delta u_{r}$ es mayor.

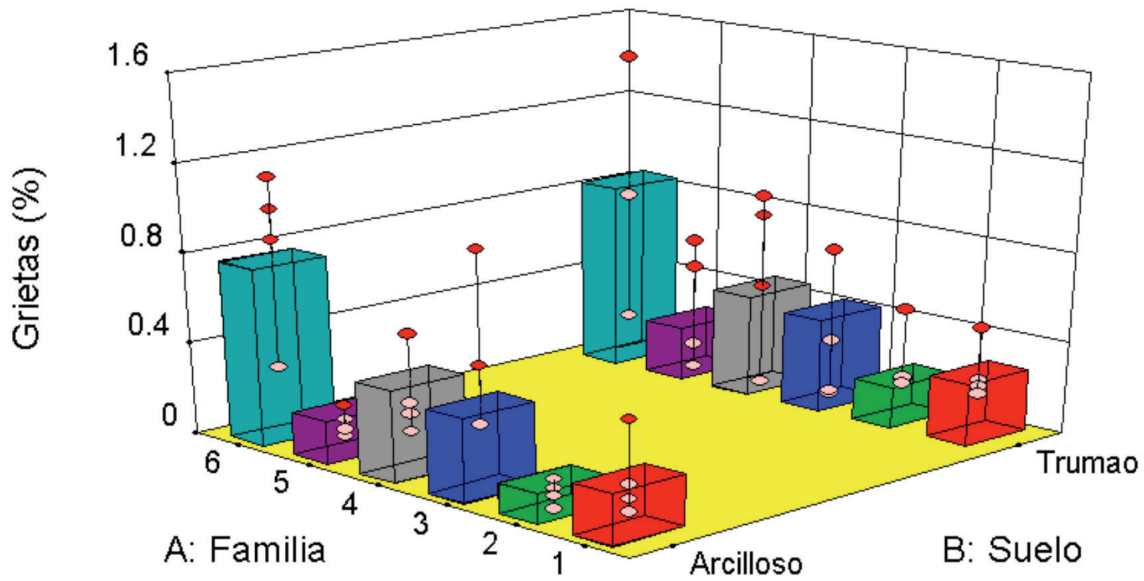

Figura 6. Comportamiento del porcentaje promedio de grietas en las semi-rodelas de las familias de Eucalyptus nitens en los diferentes suelos.

Obtenidas a $1 \mathrm{~m}, 2 \mathrm{~m}$ y $3 \mathrm{~m}$ de altura. Interacción suelo-familia, familias 1 a 6 . Suelo arcilloso y trumao. Puntos indican valores individuales.

\section{CONCLUSIONES}

Se utilizó como herramientas de segregación las características nano-mecánicas y su relación con el porcentaje de grietas medidos en volúmenes representativos del árbol. De las seis familias de Eucalyptus nitens que se estudiaron, dos de ellas presentaron menor grado de agrietamiento: la familia 2 y familia 5. Los parámetros razón de ductilidad $(E / H)$ y módulo de resiliencia $\left(u_{r}\right)$ pueden ser utilizados para la nanocaracterización de la estructura celular. Se estableció como parámetro efectivo para la segregación de familias de Eucalyptus nitens, la diferencia entre el módulo de resiliencia de la lamela media y la capa $\mathrm{S}_{2}$. Se puede relacionar este parámetro con las tensiones que se originan en la interface de la capa $\mathrm{S}_{2}$ y lamela media y con el nivel de agrietamiento de las seis familias de Eucalyptus nitens estudiadas. Además, se estableció que la lamela media es más frágil, lo que permitió relacionar este comportamiento directamente con el agrietamiento macroscópico de la madera.

\section{AGRADECIMIENTOS}

Los autores agradecen al proyecto FONDEF D07i1101 de CONICYT por el apoyo financiero y a Forestal Mininco S.A. por facilitar la materia prima para este trabajo. 


\section{BIBLIOGRAFIA}

Acevedo, A.; Bustos, C.; Lasserre, J.P.; Gacitúa, W. 2012. Efecto de la tasa de compresión en la morfología de grietas de debobinado para chapas de Eucalyptus nitens. Maderas. Ciencia y tecnología 14(3):289-301.

Baker, T.; Volker, P. 2007. Silviculture of eucalypt plantations in southern Australia for high-value solid wood products. Ciencia e Investigación Forestal 13(1):43-57.

Bolshakov, A.; Pharr, M. 1998. Influences of pileup on the measurement of mechanical properties by load and depth sensing indentation techniques. Journal of Materials Research 13(4): 1049-1058.

Bravo, S.; Espinosa, M.; Valenzuela, L.; Cancino, J.; Laserre, J.P. 2012. Efecto del raleo en el crecimiento y algunas propiedades de la madera de Eucalyptus nitens en una plantación de 15 años. Maderas. Ciencia y Tecnología 14(3):373-388.

Bustos, C.; Gacitúa, W.; Cloutier, A.; Fang, C.; Valenzuela, P. 2011. Densification of wood veneers combined with oil-heat treatment. Part III: Cell-wall mechanical properties determined by nanoindentation. BioResources 7(2): 1525-1532.

Côte, W.; Hanna, R. 1983. Ultrastructural characteristics of wood fracture surfaces. Wood Fiber Sci 15: 135-163.

Ferdinand, P.B.; Rusell E. Jr. 1992. Mechanics of material. $2^{\mathrm{a}}$ edición, McGraw-Hill Inc. New york.

Forrester, D.; Medhurst, J.; Wood, M.; Beadle C.; Valencia, J.C. 2010. Growth and physiological responses to silviculture for producing solid-wood products from Eucalyptus plantations: An Australian perspective. Forest Ecology and Management 259(9):1819-183.

Gacitúa, W.; Ballerini, A.; Laserre, J.P.; Bahr, D. 2007. Nanoindentaciones y ultraestructura en madera de Eucalyptus nitens con micro y meso fracturas. Maderas. Ciencia y Tecnología 9(3): 259-270.

Greaves, B.L.; Borralho, N.M.G.; Raymond, C.A.; Farrington, A. 1996. Use of a Pilodin for the indirect selection of basic density in Eucalyptus nitens. Canadian Journal of Forest Research 26(9): 1643-1650.

Hamilton, H.; Joyce, K.; Williams, D.; Dutkowski, G.; Potts, B. 2008. Achievements in forest tree improvement in Australia and New Zealand 9. Genetic improvement of Eucalyptus nitens in Australia. Australian Forestry 71 (2): 82-93.

Hibbeler, R.C. 2006. Mecánica de materiales. $6^{a}$ edición, Pearson educación de México S.A. México.

Hull, D.; Clyne T.W. 1996. An introduction to composite materials, $2^{\mathrm{a}}$ edition, Cambridge university Press. New York. USA.

INFOR. 2004a. Eucalyptus nitens en Chile: Procesos industriales de la madera. Informe técnico $\mathrm{N}^{0} 164$. Instituto Forestal. Valdivia, Chile. 142 p.

INFOR. 2004b. Eucalyptus nitens en Chile: Primera monografía. Informe Técnico No 164 . Instituto Forestal. Valdivia, Chile. 143 p.

Jakes, J.E.; Stone, D.S.; Frihart, C.R. 2007. Nanoindentation size effects in wood. $30^{\text {th }}$ Annual Meeting of The Adhesion Society, Tampa Bay, FL. ISSN 1086-9506.

Konnerth, J.; Gindl, W. 2006. Mechanical characterisation of wood-adhesive interphase cell walls by nanoindentation. Holzforschung 60(4): 429-433.

Leandro, L.; Ananías, R.A.; Cloutier, A.; Díaz-vaz, J.; Bermedo, M.; Sanhueza, R.; Laserre, J.P. 2008. Estudio preliminar de las grietas internas en anillos de madera inicial y su relación con características de la estructura anatómica y densidad en Eucalyptus nitens. Interciencia 33 (11): 829-834.

Medhurst, J.L.; Beadle, C.L.; Neilsen, W.A. 2001. Early-age and later-age thinning affects growth, dominance, and intraspecific competition in Eucalyptus nitens plantations. Canadian Journal of Forest Research 31(2): 187-197. 
Muñoz, F.; Valenzuela, P.; Gacitúa, W. 2012. Eucalyptus nitens: nanomechanical properties of bark and wood fibers. Applied Physics A 108(4): 1007-1014.

Nolan, G.; Greaves, B.; Washusen, R.; Parsons, M.; Jennings, S. 2005. Eucalyptus plantations for solid wood products in Australia - A review: If you don`t prune it, we can't use it. Project $n^{0}:$ PN04.3002. Forest \& Wood Products Research \& Development Corporation Victoria, Australia. 130p.

Oliver, W.C.; Pharr, G.M. 1992. An improved technique for determining hardness and elastic modulus using load and displacement sensing indentation experiments. Journal of Materials Research 7(6): 1564-1583.

Pinkard, E.; Beadle, C. 1998. Effects of green pruning on growth and stem shape of Eucalyptus nitens (Deane and Maiden) Maiden. New Forests 15(2): 107-126.

Putoczki, T.; Nair, H.; Butterfield, B.; Jackson S. 2007. Intra-ring checking in Pinus radiata D. Don: the occurrence of cell-wall fracture, cell collapse, and lignin distribution. Trees 21: 221-229.

Raymond, C. 2002. Genetics of Eucalyptus wood properties. Annals of Forest Science 59(5-6): 525-531.

Raymond, C.; Apiolaza, L. 2004. Incorporating wood quality and deployment traits in Eucalyptus globulus and Eucalyptus nitens. Plantation Forest Biotechnology for the $2{ }^{1 \text { st }}$ Century. (Ed. C. Walter and M.J. Carson) Research Signpost, Kerala. 87-99. ISBN: 81-7736-228-3.

Rezende, G.; Resende, M.; Assis, T. 2014. Eucalyptus breeding for clonal forestry. Forestry Science 81: 393-424.

Smethurst, P.; Baillie, C.; Cherry, M.; Holz, G. 2003. Fertilizer effects on LAI and growth of four Eucalyptus nitens plantations. Forest Ecology and Management 176(1-3): 531-542.

Smith, I.; Landis, E.; Gong, M. 2003. Fracture and fatigue in wood. John Wiley and Sons Southern Gate Chichester, England.

Tibbits, W.; Hodge, G. 1998. Genetics Parameters and Breeding Value Predictions for Eucalyptus nitens Wood Fiber Production Traits. Forest Science 44(4): 587-598.

Touza, M. 2001. Tensiones de Crecimiento en Eucalyptus globulus de Galicia (España). Influencia de la Silvicultura y Estrategias de Aserrado. Maderas. Ciencia y tecnología 3 (1-2): 68-89.

Valencia J.C.; Cabrera, J. 2003. Desarrollo de opciones productivas de mayor valor para plantaciones de Eucalyptus nitens en la IX y X región. Proyecto FDI/CORFO Marzo 2002-2003.

Valenzuela, P.; Bustos, C.; Laserre, J.P.; Gacitúa, W. 2012a. Fractura en madera de Eucalyptus nitens: Efecto de las propiedades mecánicas a nivel ultraestructural y de la anatomía celular. Maderas. Ciencia y tecnología 14(2): 225-238.

Valenzuela, P.; Bustos, C.; Laserre, J.P.; Gacitúa, W. 2012b. Caracterización nanomecánica de la estructura celular y anatómica de Eucalyptus nitens y su relación con la frecuencia de grietas y rajaduras en madera redonda. Maderas. Ciencia y tecnología 14(3): 321-337.

Vignote, S.; Molinero, I.; Gerard, J.; Diez, M.R. 1996. Estudio de las tensiones de crecimiento del Eucalyptus globulus Labill en Galicia y su relación con las características de la estación y morfológicas del propio árbol. Investigación Agraria: Sistemas y Recursos Forestales 5(1): 153-166.

Wimmer, R.; Lucas, B. 1997. Comparing mechanical properties of secondary wall and cell corner middle lamella in Spruce wood. IAWA Journal 18(1): 77-88.

Wu, Y.; Wang, S.; Zhou, D.; Xing, C.; Zhang, Y. 2009. Use of Nanoindentation and Silviscan to Determine the Mechanical Properties of 10 Hardwood Species. Wood and Fiber Science 41(1): 64-73.

Zou, L.; Jin, H.; Lu, W.; Li, X. 2009. Nanoscale structural and mechanical characterizacion of the cell-wall of bamboo fibers. Materials Science and Engineering: C 29 (4:) 1375-1379. 